\title{
Sarcomas de Partes Moles: Resultados do Tratamento dos Tumores de Baixo Grau
}

Soft Tissue Sarcomas: Treatment Results in Low-grade Tumors

\author{
Wilmar José Manoel', Bruno José de Queiroz Sarmento', Luiz de Paula Silveira Júnior', Deidimar Cássia Batista de Abreu', \\ Iron Pires de Abreu Neto², Erika Chaul Ferreira²
}

\section{Resumo}

Objetivos: Descrever a experiência do Serviço de Tecido Conjuntivo no tratamento dos sarcomas de baixo grau e analisar os fatores associados à recorrência local. Métodos: Setenta e seis pacientes foram analisados retrospectivamente. Informações quanto à presença de dor local, cirurgia prévia, tamanho do tumor, localização, tipo histológico, tratamento cirúrgico, recorrência e evolução foram estudados. Resultados: A sobrevida global em cinco anos foi de 94\%; a recidiva local ocorreu em 17 pacientes (22,3\%), e 9 pacientes $(11,8 \%)$ apresentaram metástases a distância. A radioterapia adjuvante foi indicada em 23 pacientes $(30,2 \%)$. Os fatores que influenciaram a recorrência local foram o tamanho do tumor $>5 \mathrm{~cm}$ (odds ratio 12,09; intervalo de confiança 95\% 1,73-84,53; $\mathrm{p}=0,012$ ) e a margem cirúrgica inadequada (odds ratio 47,16; intervalo de confiança 95\% 3,42-650,33; $\mathrm{p}=0,004$ ), identificados apenas no grupo de sarcomas de extremidades e superficiais do tronco. Conclusão: Os resultados são semelhantes aos descritos na literatura. $\mathrm{O}$ tamanho do tumor $>5 \mathrm{~cm}$ e a margem cirúrgica inadequada foram os principais fatores preditores de recorrência local. Palavras-chave: Sarcoma, Cirurgia, Radioterapia

\footnotetext{
${ }^{1}$ Serviço de Tecido Conjuntivo do Hospital Araújo Jorge da Associação de Combate ao Câncer em Goiás (HAJ-ACCG), Goiânia (GO), Brasil ${ }^{2}$ Curso de Graduação em Medicina da Universidade Federal de Goiás - Goiânia (GO), Brasil

Endereço para correspondência: Wilmar José Manoel. Hospital Araújo Jorge. Instituto de Ensino e Pesquisa. Serviço de Tecido Conjuntivo. Rua 239, no 181 - Setor Universitário - Goiânia (GO) - Brasil - CEP: 74605-070. E-mail: wilmarmanoel@brturbo.com.br
} 


\section{INTRODUÇÃO}

Os sarcomas de partes moles compõem um grupo heterogêneo de neoplasias malignas com diferentes padrões morfológicos da linhagem mesenquimal, representando cerca de $1 \%$ das neoplasias malignas em adultos. Uma outra condição rara em adultos é o sarcoma de partes moles originado da crista neural classificado como sarcoma de Ewing extra-ósseo, um integrante do grupo dos tumores neuroectodérmicos primitivos. Apesar de apresentarem um pico de incidência na infância, os sarcomas de partes moles são mais comuns na idade adulta, especialmente em maiores de 50 anos $^{1}$.

A maioria dos sarcomas de partes moles localiza-se nas extremidades, seguidos em ordem de freqüência pela cavidade abdominal, retroperitônio, parede do tronco e cabeça e pescoço ${ }^{1,2}$.

A evolução dos sarcomas de partes moles pode ser predita por alguns fatores prognósticos bem definidos, como o grau, o tipo histológico e o tamanho do tumor ${ }^{3}$. $\mathrm{O}$ estadiamento definido pelo sistema TNM da União Internacional Contra o Câncer (UICC) considera principalmente o tamanho, a profundidade, o grau histológico e a presença de metástases linfonodais ou a distância para a composição dos estádios ${ }^{4}$. Além destes fatores, a presença das margens cirúrgicas comprometidas estão relacionadas com um pior prognóstico, ${ }^{5,6}$.

Atualmente, o tratamento dos sarcomas de partes moles é norteado pelo grau histológico e pela adequação das margens cirúrgicas. Os sarcomas de baixo grau são caracterizados por uma velocidade de crescimento menor, baixo risco de metástase a distância e longa sobrevida, restando como principal objetivo do tratamento o controle local.

A base do tratamento dos sarcomas de baixo grau ainda é a ressecção com margens adequadas. Entretanto, têm-se buscado identificar pacientes com risco aumentado de recorrência e acrescentar novas estratégias que possam assegurar o controle local, como a radioterapia.

O papel da radioterapia adjuvante está bem definido nos sarcomas de alto grau com mais de $5 \mathrm{~cm}$, operados com margens comprometidas, ou menores do que $1 \mathrm{~cm}$, compondo uma proposta de cirurgia de conservação de membros ${ }^{7-9}$. Os sarcomas de alto grau $55 \mathrm{~cm}$, os sarcomas de baixo grau, os sarcomas retroperitoniais, de cabeça e pescoço, mama e ginecológico representam um ponto de controvérsia quanto à indicação da radioterapia nas suas diversas modalidades ${ }^{10-14}$.

O objetivo deste trabalho é descrever os resultados obtidos com o tratamento dos pacientes com sarcomas de baixo grau em termos de sobrevida global, controle local e fatores preditores de recidiva local, apresentando a experiência do Serviço de Tecido Conjuntivo do Hospital Araújo Jorge da Associação de Combate ao Câncer em Goiás (HAJ-ACCG) na condução desta situação clínica.

\section{METODOLOGIA}

Estudo retrospectivo envolvendo pacientes com diagnóstico histológico de sarcoma de partes moles de baixo grau, admitidos no Hospital Araújo Jorge, no período entre 1996-2000. Foram incluídos pacientes acima de 18 anos, em todos os estádios e localizações da doença.

Os dados foram extraídos do banco de dados de sarcomas do Serviço de Tecido Conjuntivo. Registraramse as informações referentes a: idade, sexo, dor local, cirurgia prévia, características histopatológicas, estadiamento, margens cirúrgicas e radioterapia adjuvante. $\mathrm{O}$ estadiamento obedeceu à versão TNM 2002 da UICC ${ }^{4}$.

Foram avaliados os seguintes fatores quanto ao risco de recorrência local: presença de dor local, tamanho do tumor $(\leq 5 \mathrm{~cm}$ ou $>5 \mathrm{~cm})$, intervenção cirúrgica prévia, grau histológico, margem cirúrgica e tipo de cirurgia. As margens de ressecção foram definidas como adequadas, se livres e com mais de $1 \mathrm{~cm}$; foram classificadas como margens inadequadas ressecçóes intralesionais, marginais, com margem comprometida ou ruptura do tumor. Considerou-se cirurgia mutiladora quando ocorreu amputação de membro ou ressecção de órgãos em monobloco, e cirurgia conservadora quando foi realizada excisão local ampla e sem ressecções de órgãos adjacentes.

Os tumores de cabeça e pescoço, mediastino, intraabdominais, retroperitônio e pelve foram denominados de sarcomas desfavoráveis, enquanto os sarcomas das extremidades e superficiais do tronco foram denominados favoráveis em relação ao tratamento cirúrgico.

A dose total de radioterapia externa no leito tumoral foi anotada em Gy. Os fatores prognósticos foram avaliados com cálculo do odds ratio e teste qui-quadrado ou teste de Fisher, quando pertinente. A regressão de Cox foi utilizada para análise multivariada. As médias de idade, tamanho do tumor e sobrevida foram comparadas com teste $t$ de Student. Para análise dos resultados do tratamento, foram excluídos pacientes no estádio IV e portadores de sarcoma de Kaposi.

A estimativa da sobrevida global se iniciou após o tratamento principal e o evento assumido foi a morte por qualquer causa. Foi considerada falência local ou distante a comprovação de lesão por exame de imagem 
e/ou anatomopatológico. Analisaram-se as curvas de sobrevida com o método de Kaplan-Meier e a diferença entre as curvas com log-rank test.

Os dados foram analisados com o software SPSS 10.0, EUA. Valores de $\mathrm{p}<0,05$ foram considerados significativos. Os pacientes assinaram o Termo de Consentimento Livre e Esclarecido, padronizado pelo Hospital Araújo Jorge, antes de se submeterem à cirurgia ou radioterapia. O estudo foi aprovado pelo Comitê de Ética da Associação de Combate ao Câncer de Goiás.

\section{RESULTADOS}

Durante o período de 1996 e 2000, foram admitidos com diagnóstico de sarcomas de partes moles 215 pacientes com mais de 18 anos. Os tumores de alto grau somaram 131 pacientes $(60,9 \%)$, e 84 casos $(39,1 \%)$ foram classificados como sarcomas de baixo grau. Os pacientes com sarcomas de baixo grau foram distribuídos em estádios, nas seguintes proporções: 24 pacientes $(28,6 \%)$ no estádio I; 54 (64,3\%) no estádio II; e 6 (7,1\%) no estádio IV. A curva de sobrevida global em cinco anos, de acordo com o grau histológico, está representada na Figura 1, sendo de $94 \%$ para sarcomas de baixo grau e $57,1 \%$ para sarcomas de alto grau $(\mathrm{p}=0,0001)$.

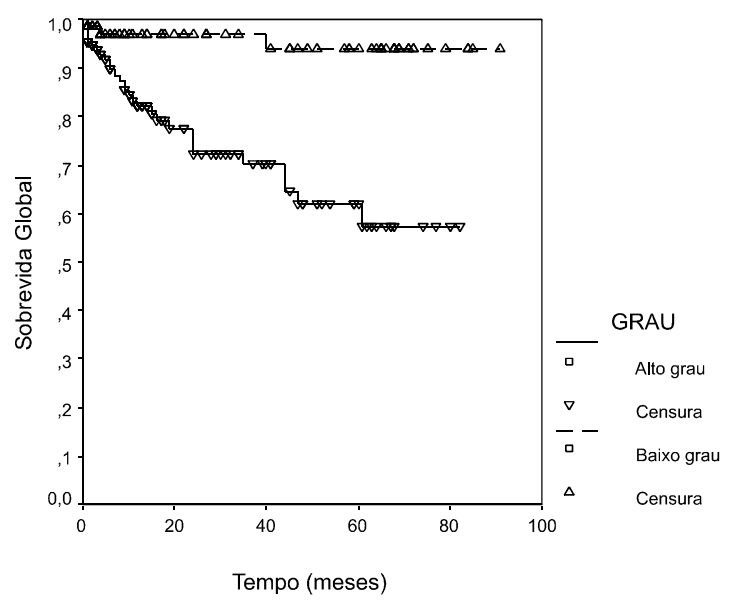

Figura 1. Curva de sobrevida global (SG) de acordo com grau histológico

As características dos pacientes com sarcomas de baixo grau (76 pacientes) estão apresentadas na Tabela 1. Foram excluídos 6 pacientes no estádio IV e 2 com informaçōes não disponíveis. A média de idade foi de 46,58 anos, variando entre 19 e 83 anos. A localização mais freqüente foi no membro inferior, ocorrendo em 27 pacientes $(35,5 \%)$. O fibrossarcoma foi o tipo histológico mais freqüente, com 25 casos $(32,8 \%) ; 18$ pacientes não tiveram a histogênese definida (23,6\%). Vinte e um pacientes $(27,6 \%)$ foram submetidos à ressecção em outras instituições. O tamanho do tumor foi maior do que $5 \mathrm{~cm}$ em $67,1 \%$ dos pacientes. A maior parte dos pacientes foi submetida à cirurgia conservadora $(59,2 \%)$ e com margens adequadas $(69,7 \%)$. A radioterapia externa adjuvante foi indicada em 23 pacientes $(30,2 \%)$. Os grupos tratados com e sem radioterapia adjuvante foram comparados por idade, sexo, localização, exame histológico, dor local, cirurgia prévia, tamanho, tipo de cirurgia e margens, não apresentando diferenças estatisticamente significantes. A dose média de radioterapia externa no leito tumoral foi de 48,4Gy, variando entre 30Gy e 60Gy.

A sobrevida média dos pacientes com sarcoma de baixo grau foi de 30,7 meses. A recorrência local foi verificada em 17 pacientes (22,3\%). Nove pacientes $(11,8 \%)$ apresentaram metástase a distância. A sobrevida média livre de recorrência local foi de 15,2 meses. Não houve diferença em recorrência local, metástase a distância, sobrevida média e sobrevida média livre de recorrência local entre os grupos, com e sem radioterapia externa (Tabela 2).

A análise univariada, incluindo as variáveis intervenções cirúrgicas prévias (biópsia ou ressecção), presença de dor local, tamanho do tumor $>5 \mathrm{~cm}$, tipo de cirurgia realizada, adequação das margens e indicação de radioterapia externa adjuvante, não identificou fatores preditores de recorrência local (Tabela 3). Nenhum fator proposto apresentou correlação independente com a recorrência local após a análise multivariada, envolvendo todos os pacientes.

O risco de recorrência local também foi avaliado, incluindo apenas os casos de sarcomas favoráveis à ressecção cirúrgica e ao tratamento radioterápico (45 pacientes). Neste grupo específico, a análise univariada não demonstrou fatores preditores de recorrência local significantes. Entretanto, a análise multivariada identificou o tamanho $>5 \mathrm{~cm}$ e a ressecção cirúrgica com margens inadequadas como fatores independentes de aumento da recorrência local (Tabela 4). Neste grupo, a dose média de radioterapia aplicada em 15 pacientes foi de 52Gy, variando entre 30Gy e 60Gy.

No outro grupo, composto por sarcomas de baixo grau desfavoráveis, nenhum fator se correlacionou com risco de recorrência local nas análises univariada e multivariada, e a dose média de radioterapia realizada em 8 pacientes foi de 40Gy, variando entre 30Gy e $60 \mathrm{~Gy}$. O papel da radioterapia adjuvante nos pacientes com margem cirúrgica inadequada não pode ser avaliado adequadamente devido ao número reduzido de pacientes nesta condição (18 pacientes). 
Tabela 1. Características dos pacientes estudados com sarcomas de baixo grau nos grupos tratados com e sem radioterapia adjuvante

\begin{tabular}{|c|c|c|c|c|}
\hline Característica & Total & $\begin{array}{c}\text { Sem } \\
\text { radioterapia } \\
n=53\end{array}$ & $\begin{array}{l}\text { Com radioterapia } \\
\qquad n=23\end{array}$ & $\mathbf{p}$ \\
\hline \multicolumn{5}{|l|}{ Sexo } \\
\hline Masculino & 30 & 22 & 8 & \multirow{2}{*}{0,58} \\
\hline Feminino & 46 & 31 & 15 & \\
\hline Idade média (anos) & 46,58 & 47,32 & 44,96 & 0,53 \\
\hline Dose total de radiação média (Gy) & & - & 48 & - \\
\hline \multicolumn{5}{|l|}{ Localização } \\
\hline Membro inferior & 27 & 20 & 7 & \multirow{8}{*}{0,16} \\
\hline Tórax & 13 & 7 & 6 & \\
\hline Intra-abdominal & 12 & 10 & 2 & \\
\hline Cabeça e pescoço & 6 & 3 & 3 & \\
\hline Pelve & 7 & 5 & 2 & \\
\hline Retroperitônio & 6 & 6 & - & \\
\hline Membro superior & 4 & 2 & 2 & \\
\hline Parede abdominal & 1 & - & 1 & \\
\hline \multicolumn{5}{|l|}{ Histologia } \\
\hline Fibrossarcoma & 25 & 19 & 6 & \multirow{8}{*}{0,54} \\
\hline Sarcoma sem especificação & 18 & 13 & 5 & \\
\hline Lipossarcoma & 15 & 8 & 7 & \\
\hline Leiomiossarcoma & 12 & 9 & 3 & \\
\hline Tumor estromal gastrintestinal & 2 & 1 & 1 & \\
\hline Histiocitoma fibroso maligno & 1 & 1 & - & \\
\hline Neurossarcoma & 1 & - & 1 & \\
\hline Outros & 2 & 2 & - & \\
\hline \multicolumn{5}{|l|}{ Biópsia prévia } \\
\hline Sim & 33 & 21 & 12 & \multirow{2}{*}{0,31} \\
\hline Não & 43 & 32 & 11 & \\
\hline \multicolumn{5}{|l|}{ Cirurgia prévia } \\
\hline Sim & 21 & 13 & 8 & \multirow{2}{*}{0,35} \\
\hline Não & 55 & 40 & 15 & \\
\hline \multicolumn{5}{|l|}{ Dor local } \\
\hline Sim & 30 & 22 & 8 & \multirow{2}{*}{0,36} \\
\hline Não & 46 & 31 & 15 & \\
\hline \multicolumn{5}{|l|}{ Tipo de cirurgia } \\
\hline Conservadora & 45 & 31 & 14 & \multirow{3}{*}{0,71} \\
\hline Mutiladora & 26 & 19 & 7 & \\
\hline Ignorado & 5 & 3 & 2 & \\
\hline \multicolumn{5}{|l|}{ Margem cirúrgica } \\
\hline Adequada & 53 & 37 & 16 & \multirow{3}{*}{0,84} \\
\hline Inadequada & 18 & 13 & 5 & \\
\hline Ignorado & 5 & 3 & 2 & \\
\hline \multicolumn{5}{|l|}{ Tamanho $>5 \mathrm{~cm}$} \\
\hline Sim & 51 & 35 & 16 & \multirow{2}{*}{0,76} \\
\hline Não & 25 & 18 & 7 & \\
\hline
\end{tabular}


Tabela 2. Resultados gerais obtidos com tratamento dos sarcomas de baixo grau

\begin{tabular}{lcccc}
\hline Resultados & $\begin{array}{c}\text { População } \\
\text { estudada } \\
\mathbf{n = 7 6}\end{array}$ & $\begin{array}{c}\text { Com } \\
\text { radioterapia } \\
\mathbf{n = 2 3}\end{array}$ & $\begin{array}{c}\text { Sem } \\
\text { radioterapia } \\
\mathbf{n = 5 3}\end{array}$ & $\mathbf{p}$ \\
\hline Recorrência local (n) & 17 & 6 & 11 & 0,60 \\
Metástases (n) & 9 & 2 & 7 & 0,65 \\
Sobrevida média (meses) & 30,7 & 40,2 & 27,4 & 0,07 \\
Sobrevida livre de recorrência local & 15,2 & 17,7 & 14,2 & 0,45 \\
média (meses) & & & & \\
\hline
\end{tabular}

Tabela 3. Análise univariada dos fatores preditores de recorrência local (76 pacientes)

\begin{tabular}{|c|c|c|c|c|c|}
\hline Variável & Sem recorrência & Com recorrência & Odds ratio & $95 \%$ IC & $\mathbf{p}$ \\
\hline \multicolumn{6}{|c|}{ Biópsia prévia } \\
\hline Não & 31 & 12 & \multirow{2}{*}{1,84} & \multirow{2}{*}{$0,72-4,71$} & \multirow{2}{*}{$0,1 \varepsilon$} \\
\hline Sim & 28 & 5 & & & \\
\hline \multicolumn{6}{|c|}{ Cirurgia prévia } \\
\hline Não & 42 & 13 & \multirow{2}{*}{1,24} & \multirow{2}{*}{$0,45-3,37$} & \multirow{2}{*}{0,6} \\
\hline Sim & 17 & 4 & & & \\
\hline \multicolumn{6}{|l|}{ Dor local } \\
\hline Não & 37 & 9 & \multirow{2}{*}{0,69} & \multirow{2}{*}{$0,30-1,59$} & \multirow{2}{*}{0,39} \\
\hline Sim & 22 & 8 & & & \\
\hline \multicolumn{6}{|c|}{ Cirurgia mutiladora } \\
\hline Não & 36 & 9 & \multirow{3}{*}{0,65} & \multirow{3}{*}{$0,28-1,47$} & \multirow{3}{*}{0,30} \\
\hline Sim & 18 & 8 & & & \\
\hline Ignorado & 5 & 0 & & & \\
\hline \multicolumn{6}{|c|}{ Margem adequada } \\
\hline Não & 13 & 5 & \multirow{3}{*}{1,22} & \multirow{3}{*}{$0,50-3,00$} & \multirow{3}{*}{0,65} \\
\hline Sim & 41 & 12 & & & \\
\hline lgnorado & 5 & 0 & & & \\
\hline \multicolumn{6}{|c|}{ Tumor $>5 \mathrm{~cm}$} \\
\hline Não & 21 & 4 & \multirow{2}{*}{0,62} & \multirow{2}{*}{$0,22-1,72$} & \multirow{2}{*}{0,35} \\
\hline Sim & 38 & 13 & & & \\
\hline \multicolumn{6}{|c|}{ Radioterapia } \\
\hline Não & 42 & 11 & \multirow{2}{*}{0,79} & \multirow{2}{*}{$0,33-1,89$} & \multirow{2}{*}{0,60} \\
\hline Sim & 17 & 6 & & & \\
\hline
\end{tabular}

Tabela 4. Análise multivariada dos fatores de recorrência local incluindo apenas sarcomas dos membros, tórax e parede abdominal (45 pacientes)

\begin{tabular}{lccc}
\hline Co-variáveis & Odds ratio & $\mathbf{9 5 \%}$ IC & p \\
\hline Dor local & 0,40 & $0,08-1,88$ & 0,24 \\
Biópsia prévia & 1,14 & $0,19-6,68$ & 0,88 \\
Cirurgia prévia & 0,62 & $0,07-5,61$ & 0,67 \\
Cirurgia conservadora & 0,43 & $0,07-2,7$ & 0,37 \\
Radioterapia adjuvante & 1,18 & $0,28-5,01$ & 0,81 \\
Tumor $>5 \mathrm{~cm}$ & 12,09 & $1,73-84,53$ & 0,012 \\
Margem inadequada & 47,16 & $3,42-650,33$ & 0,004 \\
\hline
\end{tabular}




\section{DISCUSSÃO}

O tratamento dos sarcomas de partes moles ainda constitui um desafio, mesmo nos tempos atuais. Esses tumores são considerados relativamente raros, compostos por tipos histológicos heterogêneos e apresentam comportamentos biológicos diversificados. Geralmente, os estudos clínicos que abordam o tema são realizados com grupos pequenos de pacientes alocados durante vários anos e expostos a várias estratégias de tratamento, o que tem dificultado a evolução e a padronização das condutas.

Realizou-se uma análise do tratamento dos sarcomas de partes moles de baixo grau no HAJ-ACCG e elegeramse alguns fatores para testar a associação com a recorrência local nestes 76 casos. A taxa de recidiva local obtida foi compatível com a literatura, que varia entre $13 \%$ e $37 \%$, bem como a sobrevida global, comumente superior a $87 \%$, em cinco anos ${ }^{5,6,12}$.

$\mathrm{Na}$ prática clínica, a dor tem sido valorizada como um indicador de invasão local, considerada na decisão de preservação de membro e como fator prognóstico. Apesar de ser relatada por quase $50 \%$ dos pacientes, não é um fator prognóstico consistente ${ }^{15}$. Provavelmente, os pacientes que se apresentam com dor local no momento do diagnóstico têm menor chance de preservação de membro, entretanto, os resultados oncológicos poderão ser garantidos com cirurgias radicais ou amputaçôes, atenuando o valor deste achado quando se trata de sarcoma de baixo grau.

Outro aspecto preocupante é a realização de uma intervenção cirúrgica para biópsia ou ressecção não planejada fora dos serviços oncológicos de referência. Em grandes centros, como o Memorial Sloan-Kettering Cancer Center (MSKCC), isto pode ocorrer em cerca de $33 \%$ dos casos. Um aumento significativo da recorrência local ficou bem demonstrado numa análise multivariada, envolvendo 2.084 pacientes do $M S K C C^{16}$. Pacientes operados nestas condiçóes podem apresentar um alto índice de margens comprometidas, e cerca de $46 \%$ desses pacientes apresentarão tumor residual após uma nova abordagem, o que torna a ampliação das margens um procedimento importante na obtenção do controle local adequado ${ }^{17,18}$. Nesta série, não se verificou aumento significativo da recidiva local nos pacientes submetidos a intervenções não programadas. Uma explicação para este achado é que a maioria dos pacientes apresenta sarcomas de membros e tórax (principalmente parede torácica), passíveis de cirurgia de ampliação de margens, uma conduta freqüentemente utilizada no Serviço de Tecido Conjuntivo do HAJ-ACCG.

O tamanho do tumor primário pode ser um obstáculo para o planejamento de ressecções adequadas, obtenção de margens livres e a técnica de radioterapia adjuvante. Vários trabalhos têm ressaltado que tumores com mais de $5 \mathrm{~cm}$ estão associados a um risco de recorrência local elevado, principalmente se forem maiores do que $10 \mathrm{~cm}^{5,6,16}$. Neste trabalho, verificou-se um aumento independente do risco de recorrência local nos tumores com mais de $5 \mathrm{~cm}$, localizados nos membros e tronco, confirmando o volume tumoral como um fator preditor importante de recidiva local nos sarcomas de baixo grau nestas localizações.

Com relação à discussão acerca da segurança oncológica das cirurgias de conservação de membros, verifica-se na literatura que, nos sarcomas de baixo grau, o membro pode ser salvo com ressecção ampla, reservando-se a radioterapia adjuvante para as situaçōes nas quais as margens estiverem comprometidas ou exíguas. Além disso, não há evidências de que as amputaçôes proporcionem maior sobrevida ${ }^{19,20}$. Nesta série, os procedimentos conservadores demonstraram ser oncologicamente seguros, sem aumento significativo do risco de recorrência local, o que está de acordo com a literatura.

Os sarcomas retroperitoniais e pélvicos, freqüentemente, não oferecem condições anatômicas adequadas para que uma ressecção possa ser realizada com margem adequada, sendo este o principal fator que contribui para o aumento da recorrência local ${ }^{11}$. Nestes casos, a utilização de radioterapia adjuvante tem apresentado resultados insatisfatórios e controversos, sendo limitada principalmente pela toxicidade gastrintestinal e proximidade com estruturas com baixa tolerância à irradiação, como fígado e rins, tornando o resgate radioterápico também pouco efetivo ${ }^{14}$. Neste cenário, a radioterapia intra-operatória tem sido recentemente valorizada por proporcionar uma taxa de controle local próxima de $100 \%$, desde que associada à ressecção com margem livre ${ }^{21,22}$.

O papel da margem no controle local é constantemente ressaltado em vários estudos como o principal fator relacionado ao controle local $1^{5,6,12,16}$. O objetivo do tratamento cirúrgico dos sarcomas de baixo grau deve ser, portanto, a ressecção com margens livres, com mais de $1 \mathrm{~cm}$. Considerando as experiências com braquiterapia adjuvante em sarcomas de extremidades, não há evidências de que o uso de rotina desta modalidade de irradiação possa oferecer melhor controle local ${ }^{12,18,23}$. As explicações pertinentes são o longo período de seguimento necessário para a detecção das recidivas em sarcomas de baixo grau e a relação inadequada entre a cinética celular dos sarcomas de baixo grau e o tempo de tratamento curto da braquiterapia ${ }^{12}$. Por outro lado, 
a radioterapia externa adjuvante tem se mostrado um fator importante na redução da recidiva local em pacientes operados com margens comprometidas ou com tumor residual após uma reabordagem, de acordo com o grupo do Institut Gustave Rouss ${ }^{24}$, e com o estudo retrospectivo do National Cancer Institute, Bethesda ${ }^{25}$.

Um ponto de discussão importante é a dose indicada na adjuvância. Poucos estudos abordam este tema, entretanto o estudo do Fox Chase Cancer Center demonstrou, em análise retrospectiva, que uma dose $>62,5$ Gy está relacionada com um maior controle local ${ }^{26}$. Neste estudo, a radioterapia externa adjuvante não diminuiu o risco de recorrência local. No Serviço de Tecido Conjuntivo do HAJ-ACCG, a reoperação para pacientes com margens comprometidas é uma conduta utilizada com freqüência quando o sítio do tumor é acessível, o que pode trazer controle local adequado nos sarcomas de extremidades e na parede do tronco. $\mathrm{O}$ resgate cirúrgico dos pacientes com sarcomas de localizações desfavoráveis, geralmente, não apresenta bons resultados e a radioterapia também encontra limitaçōes para um planejamento adequado e intolerância por parte dos pacientes. Nesta série, a dose de radioterapia adjuvante, em média, foi menor do que Fein et al. consideram biologicamente ativa, tanto pela toxicidade como também pelos impedimentos sociais presentes na maioria dos pacientes tratados no Hospital Araújo Jorge, ambos levando à interrupção do tratamento.

Em resumo, os pacientes com sarcomas de baixo grau, tratados no Serviço de Tecido Conjuntivo do HAJACCG, apresentam resultados semelhantes à literatura em sobrevida e controle local. Os principais fatores preditores de recidiva local foram a margem cirúrgica inadequada e o tamanho do tumor $>5 \mathrm{~cm}$, identificados na análise de subgrupo de pacientes com sarcomas de extremidades e superficiais do tronco.

\section{Potencial Conflito de Interesses: \\ Declaro não haver conflitos de interesses pertinentes.}

\section{REFERÊNCIAS}

1. Singer S, Demetri GD, Baldini EH. Management of softtissue sarcomas: An overview and update. Lancet Oncol. 2000;1:75-85.

2. Latorre MRDO, Franco EL. Epidemiologia dos sarcomas. In: Lopes A (ed). Sarcomas de partes moles. Rio de Janeiro: Medsi; 1999:3-18.

3. Vraa S, Keller J, Nielsen OS, Sneppen O, Jurik AG, Jensen OM. Prognostic factors in soft tissue sarcomas: the Aarhus experience. Eur J Cancer. 1998;34:1876-882.

4. International Union Against Cancer (UICC). TNM: classification of malignant tumors. 6th ed. New York: WileyLiss; 2002.

5. Coindre JM, Terrier P, Bui NB, Bonichon F, Collin F, Le Doussal V, et al. Prognostic factors in adult patients with locally controlled soft tissue sarcoma. A study of 546 patients from the French Federation of Cancer Centers Sarcoma Group. J Clin Oncol. 1996;14:869-77.

6. Stojadinovic A, Leung DH, Allen P, Lewis JJ, Jaques DP, Brennan MF. Primary adult soft tissue sarcoma: timedependent influence of prognostic variables. J Clin Oncol. 2002;20:4344-352.

7. Alektiar KM, Brennan MF, Singer S. Influence of site on the therapeutic ratio of adjuvant radiotherapy in soft-tissue sarcoma of the extremity. Int J Radiat Oncol Biol Phys. 2005;63(1):202-208.

8. Alektiar KM, Velasco J, Zelefsky MJ, Woodruff JM, Lewis JJ, Brennan MF. Adjuvant radiotherapy for margin-positive high-grade soft tissue sarcoma of the extremity. Int J Radiat Oncol Biol Phys. 2000;48(4):1051-1058.

9. Alekhteyar KM, Leung DH, Brennan MF, Harrison LB. The effect of combined external beam radiotherapy and brachytherapy on local control and wound complications in patients with high-grade soft tissue sarcomas of the extremity with positive microscopic margin. Int J Radiat Oncol Biol Phys. 1996;36(2):321-24.

10. Alektiar KM, Leung D, Zelefsky MJ, Brennan MF. Adjuvant radiation for stage II-B soft tissue sarcoma of the extremity. J Clin Oncol. 2002;20(6):1643-650.

11. Youssef E, Fontanesi J, Mott M, Kraut M, Lucas D, Mekhael $\mathrm{H}$, et al. Long-term outcome of combined modality therapy in retroperitoneal and deep-trunk soft-tissue sarcoma: analysis of prognostic factors. Int J Radiat Oncol Biol Phys. 2002;54(2):514-19.

12. Pisters PW, Harrison LB, WoodruffJM, Gaynor JJ, Brennan MF. A prospective randomized trial of adjuvant brachytherapy in the management of low-grade soft tissue sarcomas of the extremity and superficial trunk. J Clin Oncol. 1994;12(6):1150-155.

13. Strander H, Turesson I, Cavallin-Stahl E. A systematic overview of radiation therapy effects in soft tissue sarcomas. Acta Oncol. 2003;42(5-6):516-31.

14. Zlotecki RA, Katz TS, Morris CG, Lind DS, Hochwald $\mathrm{SN}$. Adjuvant radiation therapy for resectable retroperitoneal soft tissue sarcoma: the University of Florida experience. Am J Clin Oncol. 2005;28(3):310-16.

15. Gross JL, Younes RN, Haddad FJ, Deheinzelin D, Pinto CA, Costa ML. Soft-tissue sarcomas of the chest wall: prognostic factors. Chest. 2005;127(3):902-908.

16. Stojadinovic A, Leung DH, Hoos A, Jaques DP, Lewis JJ, Brennan MF. Analysis of the prognostic significance of microscopic margins in 2,084 localized primary adult soft tissue sarcomas. Ann Surg. 2002;235(3):424-34.

17. Davis AM, Kandel RA, Wunder JS, Unger R, Meer J, 
O'Sullivan B, et al. The impact of residual disease on local recurrence in patients treated by initial unplanned resection for soft tissue sarcoma of the extremity. J Surg Oncol. 1997;66(2):81-87.

18. Zagars GK, Ballo MT, Pisters PW, Pollock RE, Patel SR, Benjamin RS. Surgical margins and reresection in the management of patients with soft tissue sarcoma using conservative surgery and radiation therapy. Cancer. 2003;97(10):2544-553.

19. Keus RB, Rutgers EJ, Ho GH, Gortzak E, Albus-Lutter CE, Hart AA. Limb-sparing therapy of extremity soft tissue sarcomas: treatment outcome and long-term functional results. Eur J Cancer. 1994;30A(10):1459-463.

20. Williard WC, Hajdu SI, Casper ES, Brennan MF. Comparison of amputation with limb-sparing operations for adult soft tissue sarcoma of the extremity. Ann Surg. 1992;215(3):269-75.

21. Krempien R, Roeder F, Oertel S, Weitz J, Hensley FW, Timke C, et al. Intraoperative electron-beam therapy for primary and recurrent retroperitoneal soft-tissue sarcoma. Int J Radiat Oncol Biol Phys. 2006;65(3):773-79.

22. Petersen IA, Haddock MG, Donohue JH, Nagorney DM,
Grill JP, Sargent DJ, et al. Use of intraoperative electron beam radiotherapy in the management of retroperitoneal soft tissue sarcomas. Int J Radiat Oncol Biol Phys. 2002;52(2):469-75.

23. Pisters PW, Harrison LB, Leung DH, Woodruff JM, Casper ES, Brennan MF. Long-term results of a prospective randomized trial of adjuvant brachytherapy in soft tissue sarcoma. J Clin Oncol. 1996;14(3):859-68.

24. Khanfir K, Alzieu L, Terrier P, Le Pechoux C, Bonvalot S, Vanel D, et al. Does adjuvant radiation therapy increase loco-regional control after optimal resection of soft-tissue sarcoma of the extremities? Eur J Cancer. 2003;39(13):1872-880.

25. Marcus SG, Merino MJ, Glatstein E, DeLaney TF, Steinberg SM, Rosenberg SA, et al. Long-term outcome in 87 patients with low-grade soft-tissue sarcoma. Arch Surg. 1993;128(12):1336-343.

26. Fein DA, Lee WR, Lanciano RM, Corn BW, Herbert SH, Hanlon AL, et al. Management of extremity soft tissue sarcomas with limb-sparing surgery and postoperative irradiation: do total dose, overall treatment time, and the surgery-radiotherapy interval impact on local control? Int J Radiat Oncol Biol Phys. 1995;32(4):969-76.

\begin{abstract}
Objectives: The objectives were to describe the experience at our hospital's Soft Tissue Department in the treatment of low-grade sarcomas and to analyze factors associated with local recurrence. Methods: Seventy-six patients were analyzed retrospectively. Information was recorded on pain, previous surgery, localization, histopathology, surgical treatment, recurrences, and evolution. Results: Overall 5-year survival was 94\%, local failure occurred in 17 patients $(22.3 \%)$, and $9(11.8 \%)$ presented metastasis. Adjuvant radiotherapy was indicated in 23 patients (30.2\%). Factors that influenced local recurrence were tumor size $>5 \mathrm{~cm}(\mathrm{OR} 12.09 ; 95 \% \mathrm{CI} 1.73-84.53 ; \mathrm{p}=0.012)$ and inadequate margins (OR 47.16; 95\% CI 3.42-650.33; $\mathrm{p}=0.004$ ), identified only in the group with limb and superficial trunk sarcomas. Conclusions: The results were consistent with the literature. Tumor size $>5 \mathrm{~cm}$ and inadequate margins were the main factors for local recurrence.
\end{abstract}

Key words: Sarcoma, Surgery, Radiotherapy 